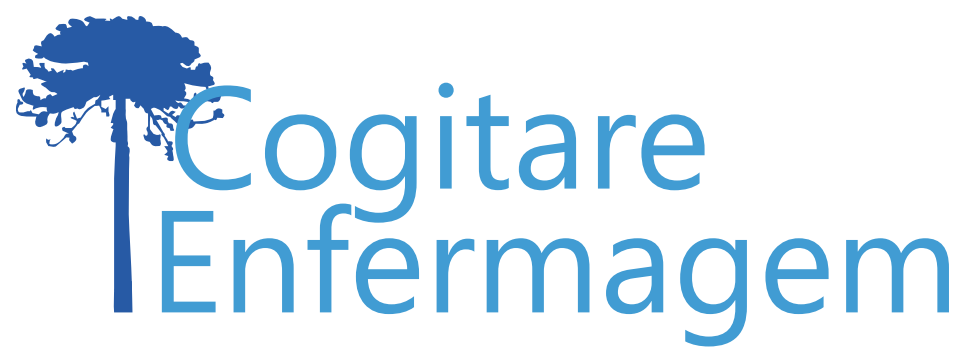

\title{
TECNOLOGIAS DO CUIDADO NA ENFERMAGEM OBSTÉTRICA: CONTRIBUIÇÃO PARA O PARTO E NASCIMENTO
}

Micheliana Rodrigues Duarte ${ }^{1}$, Valdecyr Herdy Alves², Diego Pereira Rodrigues ${ }^{3}$, Kleyde Ventura de Souza ${ }^{4}$, Audrey Vidal Pereira ${ }^{5}$, Mariana Machado Pimentel ${ }^{6}$

\section{RESUMO}

Objetivo: identificar as tecnologias do cuidado utilizadas por enfermeiras obstétricas em um Centro de Parto Normal.

Método: estudo descritivo, realizado no Centro de Parto Normal de uma maternidade do município do Rio de Janeiro, com dezoito enfermeiras obstétricas, mediante entrevistas semiestruturada entre junho e setembro de 2016. Os dados coletados foram submetidos à análise de conteúdo na modalidade temática.

Resultado: a enfermeira obstétrica utiliza métodos não farmacológicos como banho de aspersão, massagem, bola suíça, cavalinho, aromaterapia, musicoterapia, livre movimentação, ambiente acolhedor e presença do acompanhante, como práticas do seu cuidado junto às mulheres.

Conclusão: a utilização das tecnologias do cuidado permite que as enfermeiras obstétricas valorizem as práticas humanizadas no contexto do parto e nascimento.

DESCRITORES: Enfermagem Obstétrica; Obstetrícia; Parto Normal; Parto Humanizado; Saúde da Mulher.

COMO REFERENCIAR ESTE ARTIGO: Duarte MR, Alves VH, Rodrigues DP, Souza KV, Pereira AV, Pimentel MM. Tecnologias do cuidado na enfermagem obstétrica: contribuição para o parto e nascimento. Cogitare enferm. [Internet]. 2019 [acesso em "colocar data de acesso, dia, mês abreviado e ano"]; 24. Disponível em: http://dx. doi.org/10.5380/ce.v24i0.54164.

Este obra está licenciado com uma Licença Creative Commons Atribuição 4.0 Internacional.

${ }^{1}$ Enfermeira. Mestre em Saúde Materno-Infantil. Hospital da Mulher Heloneida Studart. Rio de Janeiro, RJ, Brasil. @ ${ }^{2}$ Enfermeiro. Doutor em Enfermagem. Docente de Enfermagem da Universidade Federal Fluminense. Niterói, RJ, Brasil.(? ${ }^{3}$ Enfermeiro. Mestre em Enfermagem. Docente de Enfermagem do Centro Universitário Anhanguera. Niterói, RJ, Brasil. 9 ${ }^{4}$ Enfermeira. Doutora em Enfermagem. Docente da Universidade Federal de Minas Gerais. Belo Horizonte, MG, Brasil. ${ }^{5}$ Enfermeiro. Doutor em Saúde Pública. Docente de Enfermagem da Universidade Federal Fluminense. Niterói, RJ, Brasil. ${ }^{6}$ Enfermeira. Universidade Federal Fluminense. Niterói, RJ, Brasil. () 


\title{
CARE TECHNOLOGIES IN OBSTETRIC NURSING: CONTRIBUTION FOR THE DELIVERY AND BIRTH
}

\begin{abstract}
Objective: to identify the care technologies used by obstetric nurses in a Normal Birth Center. Method: a descriptive study, performed at the Normal Birth Center of a maternity hospital in the city of Rio de Janeiro, with eighteen obstetric nurses, using semi-structured interviews between June and September 2016. The data collected were submitted to thematic type content analysis.

Result: the obstetric nurse uses non-pharmacological methods such as a spray bath, massage, Swiss ball, horse, aromatherapy, music therapy, free movement, warm environment and presence of the companion, as care practices together with the women.

Conclusion: the use of care technologies allows obstetric nurses to valorize humanized practices in the context of the delivery and birth
\end{abstract}

DESCRIPTORS: Obstetric Nursing; Obstetrics; Normal Birth; Humanized Birth; Women's Health.

\section{TECNOLOGÍAS DEL CUIDADO EN LA ENFERMERÍA OBSTÉTRICA: CONTRIBUCION PARA EL PARTO Y NACIMIENTO}

\begin{abstract}
RESUMEN:
Objetivo: identificar las tecnologías del cuidado utilizadas por enfermeras obstétricas en un Centro de Parto Normal.

Método: estudio descriptivo, realizado en el Centro de Parto Normal de una maternidad del municipio de Río de Janeiro, con dieciocho enfermeras obstétricas, mediante entrevistas semiestructuradas entre junio y septiembre de 2016. Los datos recogidos fueron sometidos al análisis de contenido en la modalidad temática.

Resultado: la enfermera obstétrica utiliza métodos no farmacológicos como baño de aspersión, masaje, bola Suiza, caballito, aromaterapia, musicoterapia, movimientos libres, ambiente acogedor y presencia de acompañante, como prácticas de cuidado a mujeres embarazadas. Conclusión: la utilización de las tecnologías del cuidado permite que las enfermeras obstétricas valoricen las prácticas humanizadas en el contexto del parto y nacimiento
\end{abstract}

DESCRIPTORES: Enfermería Obstétrica; Obstetricia; Parto Normal; Parto Humanizado; Salud de la Mujer. 
O parto, tradicionalmente, era um evento familiar, centrado na mulher e no seu protagonismo, no qual as parteiras prestavam a assistência necessária ao binômio mãe e filho no ambiente domiciliar ${ }^{(1)}$. Todavia, houve inúmeras transformações ao longo dos anos, especialmente nas últimas décadas, na atenção à mulher no ciclo gravídico-puerperal(1), influenciadas pelo modelo hospitalocêntrico, biomédico e tecnológico do parto e nascimento, despersonalizando a condição feminina, um modelo centrado na intervenção de práticas danosas, da gestação e parto considerados eventos patológicos que requeriam presença do profissional médico(1).

A inserção das boas práticas no parto normal, instituídas pela Organização Mundial de Saúde (OMS) em 1985, iniciou esse processo de desconstrução do modelo tecnológico, em prol da humanização da assistência(1). Nesse sentido, essas condutas obstétricas promovidas pela OMS propuseram a assistência baseada em evidências científicas, com base na classificação de condutas obstétricas no parto normal, segundo os critérios de utilidade, eficácia e risco. Essas recomendações originaram as seguintes categorias de práticas na assistência ao parto normal: A - práticas, demonstradamente úteis, que devem ser encorajadas; B - práticas claramente prejudiciais ou ineficazes, que devem ser eliminadas; C - práticas sem evidências para apoiar sua recomendação, devendo ser utilizadas com cautela até que novas pesquisas esclareçam a questão; D - práticas frequentemente utilizadas inadequadamente(2). Apesar dessas recomendações, as mulheres no Brasil ainda são expostas a intervenções desnecessárias ${ }^{(3)}$, sendo importante que haja empenho institucional e político para que ocorram mudanças de condutas.

A Enfermagem Obstétrica, através de seu exercício profissional(4), surge como figura central para promover a humanização da assistência e o emprego das boas práticas no parto normal, visto que promove ações que remetem ao conceito de tecnologia de cuidado(5).

A conceituação de tecnologia de cuidado classifica-se em três tipos: 1) Tecnologia leve, que implica na criação de relação entre sujeitos, por exemplo, o profissional de saúde e a cliente/usuária do sistema de saúde; 2) Tecnologia Leve-dura, que são os saberes bem estruturados que atuam no processo de saúde, como se apresenta na clínica médica e a epidemiologia; 3) Tecnologia Dura, que são os equipamentos tecnológicos que atuam junto às estruturas organizacionais ou até mesmo as máquinas, normas e rotinas de uma instituição( ${ }^{(6)}$. Nesse sentido, o conceito de tecnologia também envolve saberes e habilidades em um contexto de saber estruturado e aplicado com intencionalidade e justificativa, produzindo resultado que satisfaça as necessidades individualizadas dos seres humanos ${ }^{(7)}$.

A tecnologia no cuidado está relacionada ao desenvolvimento de práticas do processo de gestar e parir que não sejam invasivas à fisiologia do corpo feminino, à sua mente e privacidade. $O$ caráter não invasivo tem uma dimensão de estabelecimento de vínculo de confiança com a enfermeira obstétrica, e mesmo quando ocorrem condutas que expressem o cuidado na intimidade de seu corpo biológico ou sociocultural, esses não são percebidos como processo de invasão da sua privacidade ${ }^{(4-5)}$.

Assim, a enfermeira obstétrica, com a utilização de sua tecnologia de cuidado, permite promover a humanização da assistência ao parto e nascimento respeitando as mulheres, além de promover um ambiente satisfatório para o cuidado nelas centrado.

Desse modo, o estudo objetivou identificar as tecnologias do cuidado utilizadas por enfermeiras obstétricas em um Centro de Parto Normal.

\section{MÉTODO}

Estudo descritivo, exploratório, com abordagem qualitativa, realizado no Centro de Parto Normal (CPN) de uma maternidade de risco habitual, da Rede Municipal de Saúde e Defesa Civil da cidade do Rio de J̦aneiro, localizado no bairro de Bangu, que oferece atendimento às usuárias do Sistema Único de Saúde (SUS) e atende à formação de 
profissionais no campo da saúde reprodutiva.

A estrutura do CNP é composta por um CPN intra-hospitalar com sete salas individualizadas pré-parto, parto e pós-parto (PPP), um espaço de relaxamento e quatro leitos de apoio clínico, possuindo comunicação física com o Centro Cirúrgico, com três salas cirúrgicas e seis leitos de recuperação pós-anestésica. Após o parto, as puérperas e seus bebês são acomodados em um dos 61 leitos disponíveis no alojamento conjunto. 0 atendimento no CPN conta com 18 enfermeiras obstétricas em escala de trabalho de 12/60 horas obtendo-se, em média, 500 partos normais/mês em 2016, quando da investigação. Todas foram convidadas para participar do estudo.

Após aceitarem o convite e assinarem o Termo de Consentimento Livre e Esclarecido, foram-lhes assegurado o sigilo e o anonimato das entrevistas, mediante utilização de código alfanumérico: EO (de Enfermagem Obstétrica), seguido de numeral, conforme a ordem de realização da entrevista (EO1 a EO18). Foram critérios de inclusão na pesquisa: ser enfermeira obstétrica com atuação na assistência ao parto normal de risco habitual e ter um mínimo de seis meses de atuação no CPN. Foram excluídas as enfermeiras que estavam com a função de gerência ou administração na época da pesquisa.

A coleta das informações ocorreu por meio de entrevista semiestruturada, realizada de junho a setembro de 2016, em sala reservada na Instituição para este fim. Os dados foram gravados em aparelho digital, com prévia autorização das participantes transcritos integralmente e submetidos à análise de conteúdo na modalidade temática ${ }^{(8)}$, sendo utilizada a Unidade de Registro (UR) a partir da temática, como estratégia de organização do seu conteúdo. A colorimetria permitiu a identificação e o agrupamento de cada unidade, possibilitando uma visão geral da temática.

As entrevistas originaram as seguintes unidades: assistência humanizada; tecnologia não invasiva de cuidado ao parto; cuidados com o recém-nascido; acompanhante como parte do cuidado ao parto e nascimento. Essas UR fundamentaram a construção da seguinte categoria temática: 1) Tecnologias de cuidado da enfermeira obstétrica no parto e nascimento.

Atendendo à Resolução do Conselho Nacional de Saúde (CNS) n466, de 12 de dezembro de $2012^{(9)}$, o estudo foi aprovado em 04 de março de 2016 pelo Comitê de Ética em Pesquisa da Faculdade de Medicina da Universidade Federal Fluminense (UFF) sob Protocolo $n^{\circ} 1438.481 / 2016$.

\section{RESULTADOS}

As participantes expressaram que as práticas assistenciais que desenvolvem são baseadas em evidências científicas e que utilizam métodos não farmacológicos e não invasivos para o alívio da dor, como: banho de aspersão, massagem, bola suíça, banqueta meia lua, cavalinho, aromaterapia, musicoterapia, livre movimentação e/ou deambulação, penumbra e ambiente acolhedor junto ao seu acompanhante em todo o processo:

As tecnologias não farmacológicas para alívio da dor são: a liberdade dos movimentos, o uso de água morna, de movimentos pélvicos, de bolas, cavalinho, massagem, aromaterapia, a gente usa muito aqui e durante o parto a gente procura muito seguir os protocolos do Ministério da Saúde. (EO1)

A bola a gente usa principalmente junto ao banho morno e a gente costuma oferecer a bola para que ela fique mais à vontade no chuveiro. A gente coloca essa bola embaixo do chuveiro e dá um conforto maior. (EO7)

O uso dos métodos não farmacológicas do alívio da dor, que são: a bola, o cavalinho, a banqueta, o banho quente, o aromaterapia e a música, são muito favoráveis para esse processo. (EO18) 
O banho de aspersão foi o método de alívio da dor mais utilizados pelas entrevistadas durante a sua prática de atenção ao trabalho de parto e nascimento, isolado ou associado a outros métodos, sendo a forma mais evidente de relaxamento para a mulher durante o trabalho de parto e parto:

O banho de aspersão, que a gente sabe que ele regulariza as contrações, diminuiu a dor e a sensação dolorosa para essa mulher, proporciona um relaxamento melhor.(EO9)

Na nossa assistência ao parto, a gente sugere para ela outras posições, liberdade de movimentação, o banho quente. (EO15)

Foi evidenciada, também, a necessidade de instalação de uma banheira para o banho de imersão a fim de oferecer à parturiente, durante o trabalho de parto, um método de promoção de relaxamento e alívio da dor:

Segundo as evidências o que eu teria para implementar que não tem ainda seria o uso de uma banheira, que a gente não tem na unidade e que a gente sabe que tem evidências de que melhora muito o período expulsivo e o parto para a mulher e até uma tecnologia não farmacológica para o alívio da dor. (EO1)

As participantes apontaram que atuam visando minimizar o sofrimento e a dor das parturientes no processo de parir e associam os cuidados com métodos como a aromaterapia, que auxiliam no relaxamento das mulheres em todo o processo:

A gente tem o aromaterapia, a humanização que é bem forte aqui no hospital, tanto com as enfermeiras obstetras e também outros profissionais como os médicos. Muitos já estão com essa prática bastante evidenciada no atendimento à cliente.(EO3)

A gente utiliza com aroma, com óleo de canela ou óleo de lavanda, dependendo de como ela está no estado geral.(EO17)

A episiotomia foi apontada nos depoimentos das participantes:

A episiotomia pela enfermagem obstétrica tem sido zero aqui na unidade. Estamos tentando fazer com que os médicos também tenham uma redução. (EO13)

A gente já viu que proteção de períneo não diminui nem aumenta o grau de laceração, não há porque fazer a proteção perineal, assim como o óleo perineal que era muito usado, que eu aprendi ainda na minha residência não serve para nada, só para deixar lubrificado, porque se for para lacerar, vai lacerar sem óleo ou com óleo, com proteção ou sem proteção. (EO15)

Muitas práticas, como o hands-off, não manipular o bebê durante o desprendimento que a gente já viu na nossa prática e já tem evidências de que isso diminui os traumas perineais. (EO17)

Em relação aos cuidados ao recém-nascido após o parto, as participantes apontaram o contato pele a pele para o aleitamento materno na primeira hora de vida e o clampeamento tardio do cordão umbilical:

Para mim, o cuidado essencial é respeitar o bebê ao máximo, desde o momento em que ele coroa até o momento que ele realmente nasce.(EO8)

O contato no primeiro momento de vida, o contato precoce da mãe com a criança e aleitamento materno na primeira hora, visto muito a camisa do aleitamento materno.(EO17) 
O clampeamento também foi apontado pelas participantes quanto aos cuidados com o recém-nascido, em relação à dificuldade de utilização por outros profissionais de saúde:

Outra coisa que a gente viu foi do clampeamento, que passa a ser oportuno, só que apesar da gente saber da evidência científica serem benéficas para o recém-nascido, ainda tem embate com o pediatra que muitos deles, mesmo que façam o curso e saibam disso nos atrapalham nesse processo, mas a gente tenta fazer o máximo, mas tem uns que não dá para segurar. (EO7)

Historicamente tem sempre um problema com pediatras, que não gostam do clampeamento oportuno, que falam um milhão de coisas, mas a gente consegue fazer muito bem.(EO9)

As participantes destacaram a importância da presença do acompanhante no processo de cuidar do recém-nascido durante o trabalho de parto e nascimento, que favorece o sentimento de confiança das mulheres, facilitando e promovendo os seus direitos legais, tornando-as mais autônomas e satisfeitas por serem promotoras desse processo junto aos seus acompanhantes:

No momento do trabalho de parto e do parto, ou durante o nascimento do bebê a gente tem uma facilidade enorme se ela quer que o bebê seja amparado por nós, se ela quer amparar o bebê, se ela quer que o acompanhante ampare o bebê....A gente consegue aplicar isso muito bem.(EO13)

A gente consegue implementar e promover todas as práticas, as boas práticas de cuidado ao bebê junto ao acompanhante, porém depende... de cada mulher. Cada mulher demanda uma conduta, tem uma vontade própria, tem mulher que gosta de ter um acompanhante... faz questão do acompanhante ajude ...a gente não tem nenhum problema quanto a isso. (EO7)

\section{DISCUSSÃO}

A prática de cuidados da enfermeira obstétrica tem potencializado o desempenho de um exercício profissional pautado em boas práticas em obstetrícia, que priorizam o desenvolvimento de habilidades técnicas baseadas em evidências recomendadas pela OMS em detrimento de uma atenção tecnicista, que não envolva as demandas físicas, emocionais e sociais das mulheres no processo parturitivo.

As tecnologias do cuidado de enfermagem obstétrica constituem um conjunto de técnicas, procedimentos e conhecimentos utilizados na relação com a mulher, a compreensão do parto como um processo fisiológico e o respeito à integralidade corporal e psíquica ${ }^{(5,7)}$. Entretanto, essas tecnologias devem ser elaboradas e desenvolvidas por meio de práticas relacionadas ao processo de gestar e parir, que não sejam invasivas, nem interfiram no processo fisiológico do parto e do nascimento.

Desse modo, a utilização de tecnologias no cuidado à mulher com o intuito de favorecer um processo fisiológico e humanizado, é parte integrante da assistência prestada pelas participantes, pois a utilização de práticas obstétrica que valorizam a mulher e diminuem atos de intervenção mostram o avanço da enfermagem obstétrica na cidade do Rio de Janeiro.

Esse fato teve a contribuição do emprego do projeto de Implantação da Assistência de Enfermagem à Gestante e à Parturiente em duas maternidades municipais do Rio de Janeiro, com a finalidade de estabelecer práticas condizentes com o modelo humanizado de assistência ao parto(4), oportunizando à enfermagem obstétrica a legalidade institucional para promover seus cuidados e sua inserção ao contexto do parto e nascimento, mediante utilização de práticas favoráveis ao processo de nascimento, descritas anteriormente ${ }^{(7,10)}$. 
A utilização dessas tecnologias, sem dúvida, garante a segurança da mulher, além de favorecer o relaxamento para um processo mais fisiológico ${ }^{(11)}$.

Estudo realizado no município do Rio de Janeiro em uma maternidade pública apontou que as enfermeiras obstétricas perceberam que o relaxamento da mulher na água quente durante o banho de aspersão interfere na progressão do trabalho de parto: o banho auxilia a acelerá-lo já que, a partir do relaxamento, há um rápido acréscimo da dilatação do colo uterino, favorecendo uma descida mais rápida e adequada do bebê $\hat{e}^{(5)}$. Assim, o banho de aspersão é um método muito utilizado pelas participantes na assistência ao trabalho de parto e parto, favorecendo a atenção obstétrica e evitando práticas intervencionistas, desse modo, promovendo uma resposta satisfatória quanto ao relaxamento da mulher e o declínio das catecolaminas no organismo ${ }^{(12)}$.

Já o banho de imersão é classificado como um dos métodos não farmacológicos de alívio da dor durante o trabalho de parto, por proporcionar à mulher a retomada de sua autonomia no processo de parturição, pois pode mobilizar seus próprios recursos na busca de seu bem estar durante esse momento(5). Além disso, essa prática contribui para que as mulheres vivenciem o processo do parto de maneira mais harmoniosa e relaxante. Considerando que os projetos de investimentos das maternidades estão somente nos discursos políticos e institucionais(5), faz-se necessário investimento para a construção e adaptação das maternidades, seguindo os modelos para garantir a humanização da assistência.

A aromaterapia é um tratamento complementar não medicalizado, que usa os sentidos do toque e do cheiro. Cada vez mais utilizados, os óleos essenciais com aroma delicado, levemente doce, por vezes aveludado, cítrico, amadeirado, como a lavanda, o eucalipto, o jasmim, a rosa e a laranja, estão trazendo efeitos significativos na percepção da dor, na ansiedade das parturientes e, consequentemente, na duração das fases do processo parturitivo, quando a escolha bem aplicada inspira um toque de sensibilidade e sabores. Ao ser inalado, o óleo impulsiona os receptores sensitivos através do cérebro, envolvendo partes neurológicas específicas e substâncias que se harmonizam de maneira inebriante, resultando em mudanças físicas e psicológicas, capazes de obter resultados efetivos na redução da dor e da ansiedade ${ }^{(13)}$.

Sua utilização, pela enfermeira obstétrica, ocorreu juntamente com as massagens, principalmente em região lombosacra e dorsal, proporcionando o relaxamento e um cuidado humanizado, que permite atenuar a dor, a ansiedade e o medo em $89 \%$ das participantes de um estudo(14), mas não mostrou a garantia de influenciar o parto normal, pois, em um estudo de revisão sistemática, evidenciou em seus resultados que não ocorre diferença entre mulheres que utilizam a aromaterapia e as que não utilizam, para uma garantia do parto normal ${ }^{(15)}$.

As participantes apontaram que a episiotomia tem apresentado taxa zero nos indicadores e que, durante o desprendimento do polo cefálico do bebê, elas utilizam a técnica do hands-off como método alternativo para evitar as lacerações ${ }^{(3)}$. Além disso, afirmaram que na assistência ao período expulsivo, não manipulavam e não realizavam proteção na região do períneo da mulher para o desprendimento do bebê, salvo em caso de necessidade.

A propósito, um estudo realizado na Casa de Parto, em Sapopemba, São Paulo, revelou que as práticas de proteção do períneo e o manejo do polo cefálico são manobras empregadas pelas equipes de profissionais de saúde, sendo necessários mais estudos para comprovar o efeito dessas técnicas, pois uma revisão sistemática confirmou seu efeito na diminuição da taxa de episiotomia, mas não em lacerações de terceiro e quarto graus ${ }^{(16)}$.

A episiotomia é uma incisão ampliadora do orifício vulvar realizado na região do corpo perineal. É uma das práticas que mais tem causado morbidades maternas durante o pós-parto, por predispor a mulher ao aumento de perda sanguínea, infecção puerperal, disfunção sexual como a dispareunia, incontinência urinária, prolapso vaginal, entre outras alterações, quando comparada com outros tipos de trauma perineal. Cabe ressaltar que a episiotomia é um dos únicos procedimentos realizados sem qualquer consentimento prévio 
da paciente ${ }^{(16-17)}$, perpetuando um modelo intervencionista e tecnocrático no cuidado da mulher, e não reduzindo os riscos de trauma perineal(18).

Desse modo, no modelo de humanização, torna-se claro que os ambientes em que a mulher é inserida no período gravídico-puerperal são importantes, cabendo aos profissionais que as assistem valorizar mais os aspectos fisiológicos do gestar e parir, evitando intervenções desnecessárias e garantindo um processo de trabalho qualificado e seguro para a mulher e seu bebê.

Observou-se o quanto são importantes, para as participantes, os cuidados imediatos e as práticas exercidas na assistência ao recém-nascido, na sua primeira hora de vida, ressaltando-se que essas ações estão baseadas em evidências científicas. A OMS recomenda colocar os bebês em contato pele a pele com suas mães imediatamente após o parto, durante pelo menos uma hora, encorajando as mães a reconhecerem quando seus bebês estão prontos para mamar, oferecendo ajuda, se necessário. O apoio para que o início do aleitamento se dê nesse período sensível, em que mãe e recém-nato estão alertas, corresponde ao Passo 4 da Iniciativa Hospital Amigo da Criança, sendo uma prática que pode reduzir a mortalidade neonatal, porque quanto mais se prorroga o início, maiores as chances de mortalidade neonatal causadas por infecções ${ }^{(19)}$.

O clampeamento tardio do cordão umbilical, conforme a OMS, é uma forte recomendação para todos os nascimentos, devendo ser realizado aproximadamente entre um e três minutos após o nascimento, iniciando concomitantemente os primeiros cuidados ao recém-nascido. No entanto, atualmente, a cobertura dessa intervenção tem sido limitada, devido à falta de informações sobre seus benefícios, bem como em função de preocupações suscitadas a respeito da prática ${ }^{(20)}$. Dessa forma, o clampeamento tardio do cordão umbilical deve ser propagado e estimulado para promover a saúde do recémnascido.

O depoimento das participantes permitiu compreender nos cuidados de atenção ao recém-nascido por esses profissionais que houve priorização do modelo tecnocrático com a separação entre a mãe e o bebê. Isso ocorre porque a lógica da assistência hospitalar é baseada na produção de procedimentos ${ }^{(21)}$. Dessa forma, as práticas de atenção ao recémnascido ocorrem de forma acelerada e mecânica para não atrapalhar o papel dos demais profissionais que atuam na sala de parto.

As participantes destacaram a importância do apoio do profissional de saúde em relação à mulher ao promover segurança, atenção nos cuidados junto ao acompanhante, assim como estimular e promover o seu direito para que ela seja respeitada durante todo 0 processo de parto e nascimento. Assim, a presença do acompanhante durante o trabalho de parto, parto e nos cuidados imediatos ao recém-nascido, proporcionou bem estar físico e emocional à mulher. Entretanto, respeitando esse momento único da mulher e seu concepto, devem-se priorizar os princípios da humanização do parto e nascimento, favorecendo a singularidade, seu significado e a presença do acompanhante.

A Lei $n^{\circ} 11.108$, de 7 de abril de 2005, instituiu nos serviços de saúde do Sistema Único de Saúde ou nos conveniados, a obrigatoriedade da presença de um acompanhante de livre escolha da mulher durante o período de pré-parto, parto e puerpério imediato(22). Portanto, a mudança de modelo na parturição, com o conhecimento da mulher e a inserção do acompanhante na cena de apoio e cuidado, fazem-se necessários como emprego das boas práticas no cuidado à saúde da mulher.

O estudo realizado apresentou como limitação o número dos participantes, não permitindo uma extrapolação dos resultados, pois não pegou toda a população. Sugerese, portanto, futuras investigações utilizando um número ampliado de enfermeiros da unidade, para abranger a totalidade do cenário. 
A centralidade do cuidado à mulher é uma premissa descrita nas melhores evidências cientificas, além de norteadas pelas políticas públicas de saúde, cabendo à enfermeira obstétrica, com sua prática assistencial, reafirmar o respeito à fisiologia do parto, através da utilização das práticas não farmacológicas e não invasivas no processo de parturição, como banho de aspersão, massagem, bola suíça, banqueta meia lua, cavalinho, aromaterapia, musicoterapia, livre movimentação e/ou deambulação, penumbra e um ambiente acolhedor. As tecnologias do cuidado aqui descritas contribuem para a promoção do parto respeitoso, favorecendo o protagonismo da mulher.

Conclui-se, portanto, que as tecnologias utilizadas pelas enfermarias obstétricas no campo do parto e nascimento podem favorecer a autonomia das mulheres no processo de parturição, porém, devem ser ofertadas para cada mulher como opção de cuidado, e não como uma prática impositiva às parturientes, que necessitam ser orientadas e esclarecidas a participarem ativamente do ato de parir, desde o pré-natal. Sem dúvida, a prática da enfermeira obstétrica permeia a valorização da sua autonomia, mostrando a sua importância para a garantia de emprego de práticas para um cuidado centrado nas necessidades da mulher.

\section{REFERÊNCIAS}

1. Melo BM, Gomes LBS, Henriques ACPT, Lima SKM, Damasceno AKC. Implementação das boas práticas na atenção ao parto em maternidade de referência. Rev. RENE. [Internet]. 2017 [acesso em 2017 jul 22]; 18(3). Disponível em: http://periodicos.ufc.br/rene/article/view/20065.

2. Vogt SE, Silva KS, Dias MAB. Comparação de modelos de assistência ao parto em hospitais públicos. Rev. Saúde Públ. [Internet]. 2014 [acesso em 2017 jul 22]; 48(2). Disponível em: http://dx.doi.org/10.1590/ S0034-8910.2014048004633.

3. Côrtes CT, Santos CS, Caroci AS, Oliveira SG, Oliveira SMJV, Riesco MLG. Metodologia de implementação de práticas baseadas em evidências científicas na assistência ao parto normal: estudo piloto. Rev. Essc. Enferm. USP. [Internet]. 2015 [acesso em 2017 jul 22]; 49(5). Disponível em: http://dx.doi. org/10.1590/S0080-623420150000500002.

4. Camacho KG, Progiant JM. A transformação da prática obstétrica das enfermeiras na assistência ao parto humanizado. Rev. Eletr. Enferm. [Internet]. 2013 [acesso em 2017 jul 22]; 15(3). Disponível em: https://doi.org/10.5216/ree.v15i3.18588.

5. Prata JA, Progiant JM. A influência da prática das enfermeiras obstétricas na construção de uma nova demanda social. Rev. enferm. UERJ. [Internet]. 2013 [acesso em 2017 jul 22]; 21(1). Disponível em: http:// www.e-publicacoes.uerj.br/index.php/enfermagemuerj/article/view/6341/4516.

6. Salvador PTCO, Oliveira RKM, Costa TD, Santos VEP, Tourinho FSV. Tecnologia e inovação para o cuidado em enfermagem. Rev. enferm. UERJ. [Internet]. 2012 [acesso em 2017 jul 22]; 20(1). Disponível em: http://www.e-publicacoes.uerj.br/index.php/enfermagemuerj/article/view/4004.

7. Takemoto AY, Corso MR. Parto humanizado e a assistência de enfermagem: uma revisão da literatura. Arq. ciências saúde UNIPAR [Internet]. 2013 [acesso em 2017 jul 22];17(2). Disponível em: http://revistas. unipar.br/index.php/saude/article/view/5002.

8. Câmara RH. Análise de Conteúdo: da teoria à prática em pesquisas sociais aplicadas às organizações. Gerais. [Internet]. 2013 [acesso em 2017 jul 22]; 6(2). Disponível em: http://www.fafich.ufmg.br/gerais/ index.php/gerais/article/view/306.

9. Ministério da Saúde (BR). Conselho Nacional de Saúde. Diretrizes e normas regulamentadoras de pesquisa envolvendo seres humanos. Resolução n. 466, de 12 de dezembro de 2012. Brasília; 2012.

10. Ministério da Saúde (BR). Secretaria de Ciência, Tecnologia e Insumos Estratégicos. Departamento de Gestão e Incorporação de Tecnologias em Saúde. Diretrizes nacionais de assistência ao parto normal. [Internet]. Brasília: Ministério da Saúde; 2017 [acesso em 2017 jul 22]. Disponível em: http://bvsms.saude. 
11. Guida NFB, Lima GPV, Pereira ALF. O ambiente de relaxamento para humanização do cuidado ao parto hospitalar. REME. [Internet]. 2013 [acesso em 2017 jul 22]; 17(3). Disponível: http://www.reme.org. br/artigo/detalhes/670.

12. Barbieri M, Henrique AJ, Chors FM, Maia NL, Gabrielloni MC. Banho quente de aspersão, exercícios perineais com bola suíça e dor no trabalho de parto. Acta Paul. Enferm. [Internet]. 2013 [acesso em 2017 jul 22]; 26(5). Disponível em: http://dx.doi.org/10.1590/S0103-21002013000500012.

13. Kaviani M, Machbool S, Azima S, Tabael MH. Comparison of the effect of aromatherapy with Jasminum officinale and Salvia officinale on pain severity and labor outcome in nulliparous women. Iran J. Nurs. Midwifery Res. [Internet]. 2014 [acesso em 2017 jul 22]; 19(6). Disponível em: https://www.ncbi.nlm. nih.gov/pmc/articles/PMC4280734/.

14. Gayeski ME, Bruggemann OM. Métodos não farmacológicos para alívio da dor no trabalho de parto: uma revisão sistemática. Texto contexto - enferm. [Internet]. 2010 [acesso em 2018 maio 23]; 19(4). Disponível em: http://dx.doi.org/10.1590/S0104-07072010000400022.

15. Osório SMB, Junior LGS, Nicolau AIO. Avaliação da efetividade de métodos não farmacológicos no alívio da dor do parto. Rev. RENE. [Internet]. 2014 [acesso em 2018 maio 23]; 15(1). Disponível em: http:// www.periodicos.ufc.br/rene/article/view/3112/2386.

16. Silva FMB, Paixão TCR, Oliveira SMJV, Leite JS, Riesco MLG, Osava RH. Assistência em um centro de parto segundo as recomendações da Organização Mundial da Saúde. Rev. Esc. Enferm. USP. [Internet]. 2013 [acesso em 2017 jul 22 jul]; 47(5). Disponível em: http://dx.doi.org/10.1590/S0080623420130000500004.

17. Dengo VAR, Silva RS, Souza SRRK, Aldrighi JD, Wall ML, Cancela FZV. Aepisiotomia na percepção de puérperas. Cogitare enferm. [Internet]. 2016 [acesso em 2017 jul 22]; 21(3). Disponível em: http://dx. doi. org/10.5380/ce.v21i3.44060.

18. Sauaia ASS, Serra MCM. Uma dor além do parto: violência obstétrica em foco. Revista de direitos humanos e efetividade. [Internet]. 2016 [acesso em 2017 jul 22]; 2(1). Disponível em: http://www.indexlaw. org/index.php/revistadhe/article/view/1076.

19. Boccolini CS, Carvalho ML, Oliveira MIC, Pérez-Escamilla R. Breastfeeding during the first hour of life and neonatal mortality. J pediatr. [Internet]. 2013 [acesso em 2017 jul 22]; 89(2). Disponível em: http:// dx.doi.org/10.1016/j.jped.2013.03.005.

20. Organização Mundial de Saúde. Recommendations for the prevention and treatment of postpartum haemorrhage: evidence base. [Internet] Geneva: WHO; 2012 [acesso em 2017 jul 22]. Disponível em: http://apps.who.int/iris/bitstream/handle/10665/75411/9789241548502 eng. pdf;jsessionid=5DE5140EF13DF1850318A05488AD7F02? sequence=1.

21. Santos LM, da Silva JCR, Carvalho ESS, Carneiro AJS, de Santana RCB, Fonseca MCC. Vivenciando o contato pele a pele com o recém-nascido no pós-parto como um ato mecânico. Rev. bras. enferm. [Internet]. 2014 [acesso em 2017 jul 22]; 67(2). Disponível em: http://dx.doi.org/10.5935/00347167.20140026.

22. Brüggemann OM, Ebsen ES, Oliveira ME, Gorayeb MK, Ebele RR. Motivos que levam os serviços de saúde a não permitirem acompanhante de parto: discursos de enfermeiros. Texto contextoenferm. [Internet]. 2014 [acesso em 2017 jul 22]; 23(2). Disponível em: http://dx.doi.org/10.1590/010407072014002860013. 
Autor Correspondente:

Diego Pereira Rodrigues

Centro Universitário Anhanguera

R. Desembargador Leopoldo Muylaert, 307 - 24350-450 - Niterói, RJ, Brasil

E-mail: diego.pereira.rodrigues@gmail.com

\section{Contribuição dos autores:}

Contribuições substanciais para a concepção ou desenho do estudo; ou a aquisição, análise ou interpretação de dados do estudo - MRD, VHA, DPR, AVD, MMP

Elaboração e revisão crítica do conteúdo intelectual do estudo - MRD, VHA, DPR, KVS, AVD, MMP

Aprovação da versão final do estudo a ser publicado - MRD, VHA, DPR, KVS, AVD, MMP

Responsável por todos os aspectos do estudo, assegurando as questões de precisão ou integridade de qualquer parte do estudo - MRD, VHA, DPR, KVS, AVD, MMP 